\title{
The effects of valid and invalid expectations about stimulus valence on behavioural and electrophysiological responses to emotional pictures
}

\author{
Johnen, A-K., \& Harrison, N.R.
}

Department of Psychology, Liverpool Hope University, Liverpool, UK

Corresponding Author:

Dr Neil Harrison

Department of Psychology

Liverpool Hope University

Liverpool

UK

L16 9JD

Email address: harrisn@hope.ac.uk 


\begin{abstract}
Previous studies have shown that uncertainty about the expected valence of an upcoming stimulus modulates behavioural and neural responses to the stimulus. However, relatively little is known about how invalid emotion expectations (i.e. expecting a positive picture but seeing a negative picture) affect neural and behavioural responses. To investigate this, two experiments were conducted where participants viewed and rated the pleasantness of emotional pictures that were preceded by a cue. In Experiment 1, there were three cue conditions: uncertain, valid and invalid. The uncertain cue did not indicate the valence of the upcoming picture, whereas cues in the valid condition (70\% of positive and negative cues) correctly indicated the valence of the upcoming picture. The remaining trials were invalid, where the valence of the picture differed from the expected valence. Behavioural results showed that invalidly cued negative pictures elicited more neutral valence ratings compared to validly cued pictures. In Experiment 2 we replicated the findings of Experiment 1, and in addition found an increased amplitude for the early $(400-600 \mathrm{~ms})$ portion of the late positive potential (LPP) for invalidly compared to validly cued pictures. Together, these results show that invalid expectations influence the neural and behavioural processing of subsequently presented emotional pictures, where invalidly cued pictures led to attenuated (i.e., more neutral) emotional responses, and enhanced early LPP amplitude, compared to validly cued pictures.
\end{abstract}




\section{Introduction}

In daily life we normally experience a wide variety of emotions in response to many different situations and events. In many cases these emotional reactions are anticipated in advance, such as the feeling of joy at the birth of a baby, or the grief of losing a loved one. However, quite often emotions are not anticipated in advance and are therefore unexpected. In this context, the term unexpected can have several different meanings. For example, it can refer to cases where an emotionally arousing event occurs without any prior warning, such as suddenly seeing an accident. It can also mean that an upcoming emotional event is expected, but the valence of the emotional response is unknown beforehand (i.e., 'expectation uncertainty'), for example as often occurs during gambling (Mellers, Schwartz, Ho, Ritov, 1997). The term 'unexpected' can also refer to situations where an event of a specific emotional valence is anticipated, but the outcome elicits an emotion of a different valence, i.e., when one expects an event to turn out well, but instead it turns out badly (for example one expects to pass an exam, but instead one fails it). Understanding how these invalid/ incongruent expectations (i.e., where there is a discrepancy between expected and actual emotional outcomes) affect processing of a stimulus is important, as outside the lab very few expectations are guaranteed to be correct.

Most previous research into the effects of expectations on emotional processing has tended to focus on situations where the valence of an upcoming stimulus is indicated in advance by a cue (i.e., 'expectation certainty'), compared to cases where the valence of the upcoming stimulus is not indicated in advance (i.e., 'expectation uncertainty'). For example, Lin et al. (2012) showed that under conditions of expectation certainty the emotional impact of visual scenes was enhanced (as measured by more negative valence ratings), compared to under conditions of expectation uncertainty. In addition to investigating behavioural 
responses, electrophysiological responses can provide additional understanding of the influence of expectations on emotions, especially as regards the time-course of neural processing. Event-related potentials (ERPs), which, with their temporal resolution in the millisecond range, are extremely useful in this context due to the rapid speed with which the initial processing of emotional stimuli generally unfolds.

Indeed, previous research has uncovered a number of ERP components occurring shortly after presentation of a stimulus that are sensitive to emotional meaning (Foti, Hajcak, \& Dien, 2009). Of particular relevance here are the $\mathrm{P} 2$, the $\mathrm{N} 2$, and the late positive potential (LPP). The P2, a positive deflection peaking around $150 \mathrm{~ms}$ after picture onset at frontocentral sites, is thought to be associated with feature detection (Lin, Liang, Jin, \& Zhao, 2018; Olofsson, Nordin, Sequeira, \& Polich, 2008). Following the P2 is the N2, a negative deflection in the ERP maximal around $250 \mathrm{~ms}$ after picture onset, which is considered to be related to stimulus classification and discrimination (Lin et al., 2018; Olofsson et al., 2008). The P300/slow wave evoked by emotionally arousing images, commonly referred to as the late positive potential (LPP), is a positive deflection in the ERP that is maximal over posterior-superior sites, beginning as early as $300 \mathrm{~ms}$ after picture onset (Foti et al., 2009; Olofsson et al., 2008). The early part of the LPP is thought to be modulated by arousal, taskrelevance, and stimulus predictability, and the later part of the LPP to reflect more in-depth, controlled processing, of emotional pictures (Gole et al., 2012; Leutgeb, Schäfer, \& Schienle, 2009; Olofsson et al., 2008).

Several studies have reported that viewing pictures under conditions of expectation certainty was associated with an increased LPP compared to under conditions of expectation uncertainty (Gole, Schäfer, \& Schienle, 2012; Lin et al., 2012; Lin, Jin, et al., 2015). In contrast, Dieterich, Endrass, and Kathmann $(2016$; 2017) reported greater LPP amplitude in 
the uncertain compared to the certain expectation condition. The apparent inconsistency in the results between different studies may be attributed to the different types of anticipations involved. While some research used an implicit anticipation paradigm (Gole et al., 2012; Lin et al., 2012), instead Dietrich et al. $(2016,2017)$ employed an explicit anticipation paradigm, where participants were asked to indicate the upcoming emotion in terms of its likelihood. This may have focused participants' attention toward resolving the discrepancy between the expected and presented emotion, whereas an implicit style of anticipation may have focused attention more toward those stimuli where the emotional content was known in advance (Lin et al., 2018). Furthermore, results regarding the P2 and N2 ERP components have been mixed, with some studies reporting increased P2 and N2 amplitudes following expectation certainty (Lin et al., 2012), while others showed an increased P2 and N2 amplitude following expectation uncertainty (Gole et al., 2012). Other studies reported no effects of expectation on either the P2 or N2 (Lin et al., 2018). Notwithstanding the mixed nature of the findings in these studies, nevertheless together they show that expectations (in particular, expectation certainty vs uncertainty) about the valence of an upcoming stimulus can influence the processing of the stimulus, as reflected by both behavioural and ERP measures.

Somewhat surprisingly, there has been less research into the effects of invalid expectations on the processing of emotional pictures, i.e., cases where the valence of a stimulus is different from its expected valence. According to the "costs and benefits of expectations' theory, invalid expectations should amplify the emotional response to an event, whereas valid expectations should attenuate affective reactions (Golub, Gilbert, Wilson, 2009). To the best of our knowledge, the only study investigating the effects of invalid expectations on the processing of emotional pictures was conducted by Osman, Paczynski and Jha (2017). Their study, using a sample of military personnel preparing for deployment, investigated how invalid expectations affected the neural processing of neutral and combat- 
related negative pictures. Cues were invalid in $25 \%$ of trials, and participants were not informed about the presence of invalid cues prior to the experiment. Results showed that LPP amplitudes at centro-parietal and anterior sites were greater for pictures that were preceded by a negative cue compared to those preceded by a neutral cue, but they reported no effects of cue validity on LPP amplitudes. Typically the P3/LPP amplitude is more positive for invalidly compared to validly cued stimuli, in both affective (Lin, Schulz, \& Straube, 2015, 2016) and non-affective (e.g., spatial) cueing paradigms (Curran, Hills, Patterson, \& Strauss, 2001; Mangun \& Hillyard, 1991). In the studies by Lin, Schulz \& Traube $(2015,2016)$, a cue predicted either a neutral or a fearful facial expression; the cues correctly predicted the facial expressions in $75 \%$ of trials (expectation congruency trials), while in the remaining trials the cues incorrectly predicted the facial expressions (expectation incongruent trials). The P3/LPP amplitude was larger in the incongruent versus congruent trials, consistent with research showing an increased P3/LPP in response to emotional stimuli that were unpredictable (Delplanque et al., 2005; Volpe et al., 2007).

Instead of generating expectations of neutral and negative images, in the current study the cues were designed to create expectations of either positive or negative images. Subsequently, in the invalid conditions there would be a large valence difference between the expected and the actual image (i.e., positive to negative, or negative to positive valence). Thus far, the effects of expectation violations, where the subsequently presented picture has an opposite valence from that which was expected, remain unknown. This is an important gap in our understanding, as this variety of expectation violation occurs regularly in everyday life, for example when an encounter with an expected positive valence (e.g., watching a film, meeting a friend, viewing a work of art, and so on) turns out to instead elicit a negative emotional outcome. 
The aim of the current study was therefore to address the main limitations of previous research on the effects of valid and invalid expectations on the processing of emotional pictures. Specifically, using an affective-cueing design, we wanted to investigate the effects of emotion expectation violations when the presented stimulus had an opposite valence to the expected valence. Experiment 1 aimed to test how invalid expectations affected valence ratings of emotional pictures, as previous studies of affective expectation violation did not examine participants' ratings of the images (Osman et al., 2017). Experiment 2 investigated ERP responses in addition to the self-report ratings, to investigate the electrophysiological processes related to affective cue validity. Only female participants were tested, in line with previous studies (e.g., Gole et al., 2012), as there are well-known differences in emotional processing between males and females (e.g., Lin et al., 2014).

\section{Experiment 1}

The aim of Experiment 1 was to investigate the effects of valid and invalid expectations on valence ratings for positive and negative pictures using an affective-cueing design. In Experiment 1 there were three cue conditions: uncertain, valid and invalid. The uncertain cue did not indicate the valence of the upcoming picture. In the valid condition (70\% of positive and negative cues) the cue correctly indicated the valence of the upcoming picture. The remaining trials were invalid, where the valence of the picture differed from the expected valence. We predicted that participants would rate invalidly cued negative pictures as less negative compared to validly cued negative pictures. For positive images, we expected the reverse, i.e., that invalidly cued positive pictures would lead to lower valence ratings compared to validly cued positive pictures. Further, we expected that uncertainly cued negative pictures would lead to with higher valence ratings compared to validly cued 
negative pictures, in line with the findings of Lin et al. (2012). Finally, we predicted that participants would be slower to respond in the invalid conditions.

\section{Method}

\section{Participants}

Twenty-four female participants (age: $M=21.9 ; S D=4.0$ years) took part in this experiment. All participants reported that they were right handed and had normal or corrected-to-normal vision. The study was conducted in accordance with the standard ethical guidelines as defined in the Declaration of Helsinki and written informed consent was obtained prior to the testing. The study was approved by the Ethics Committee of Liverpool Hope University.

\section{Stimuli}

Three-hundred and thirty pictures from the International Affective Picture System (IAPS; Lang, Bradley, \& Cuthbert, 2008) were selected and placed into the positive or negative emotion category based on valence rating norms. For inclusion into the negative valence category pictures had to have a valence rating norm of less than 4 (valence: $M=2.54$, $S D=0.72$; arousal: $M=5.76, S D=5.76$ ) and for the positive category a valence rating norm of 6.5 or greater (valence: $M=7.43, S D=0.54$; arousal: $M=4.54, S D=0.93$ ). Both categories were matched for the number of pictures that depicted faces. Thirty of these pictures (15 positive and 15 negative) were selected for inclusion in a practice block. The remaining 300 images were included in the main experiment. The two categories differed significantly in valence $t(164)=70.67, p<.001$, and arousal $t(164)=12.89, p<.001$. Each picture was only presented once during the experiment, and all pictures were matched for luminance and size (on-screen display size: 512 x 384 pixels). 


\section{Procedure}

Participants were seated approximately $80 \mathrm{~cm}$ from a monitor in a dimly lit room.

After giving informed consent, participants were informed that they would be presented with emotional pictures that were always preceded by a cue. A plus sign ('+') indicated an upcoming positive picture, a minus sign ('-') indicated a negative picture, and a question mark ('?') indicated that the following picture could be either positive or negative.

Participants were asked to rate the pleasantness of the images on a 9-point Likert-type scale after each trial $(1=$ extremely unpleasant; $9=$ extremely pleasant $)$. The numbers of trials per condition are shown in Table 1.

Table 1 Number of trials per cue and picture condition

\begin{tabular}{lccc}
\hline \multicolumn{3}{c}{ Cue } \\
\hline Picture & + & $?$ & - \\
\hline Positive & Valid (70 trials) & Uncertain (50 trials) & Invalid (30 trials) \\
Negative & Invalid (30 trials) & Uncertain (50 trials) & Valid (70 trials) \\
\hline
\end{tabular}

Participants first undertook a practice block with 10 trials of the valid conditions. In the main experiment, on each trial participants were again presented with a cue followed by an emotional picture. $30 \%$ of the positive and $30 \%$ of the negative pictures were preceded by an invalid cue meaning that a plus sign was followed by a negative picture or that a minus sign was followed by a positive picture. For the uncertain cue condition, $50 \%$ of cues were followed by positive pictures and $50 \%$ by negative pictures.

Each trial started with the presentation of a black fixation cross for $500 \mathrm{~ms}$ followed by a blank screen for between 600 to $1000 \mathrm{~ms}(M=800 \mathrm{~ms})$. The cue was presented for 200 ms followed by another blank screen for between 1600 to $2000 \mathrm{~ms}(M=1800 \mathrm{~ms})$. After picture presentation (1000 ms duration), a blank screen was presented for $200 \mathrm{~ms}$. The rating 
screen was then presented until the participant responded, or for a maximum of $2000 \mathrm{~ms}$.

This was followed by another blank screen for $200 \mathrm{~ms}$ before the next trial began (see Fig. 1).

The study contained five blocks of 6 minutes, and took approximately 30 minutes overall. Participants were able to rest after each block for as long as they wished. E-Prime 2.0 (Psychology Software Tools, Pittsburgh, PA) was used to present stimuli and collect behavioural responses.

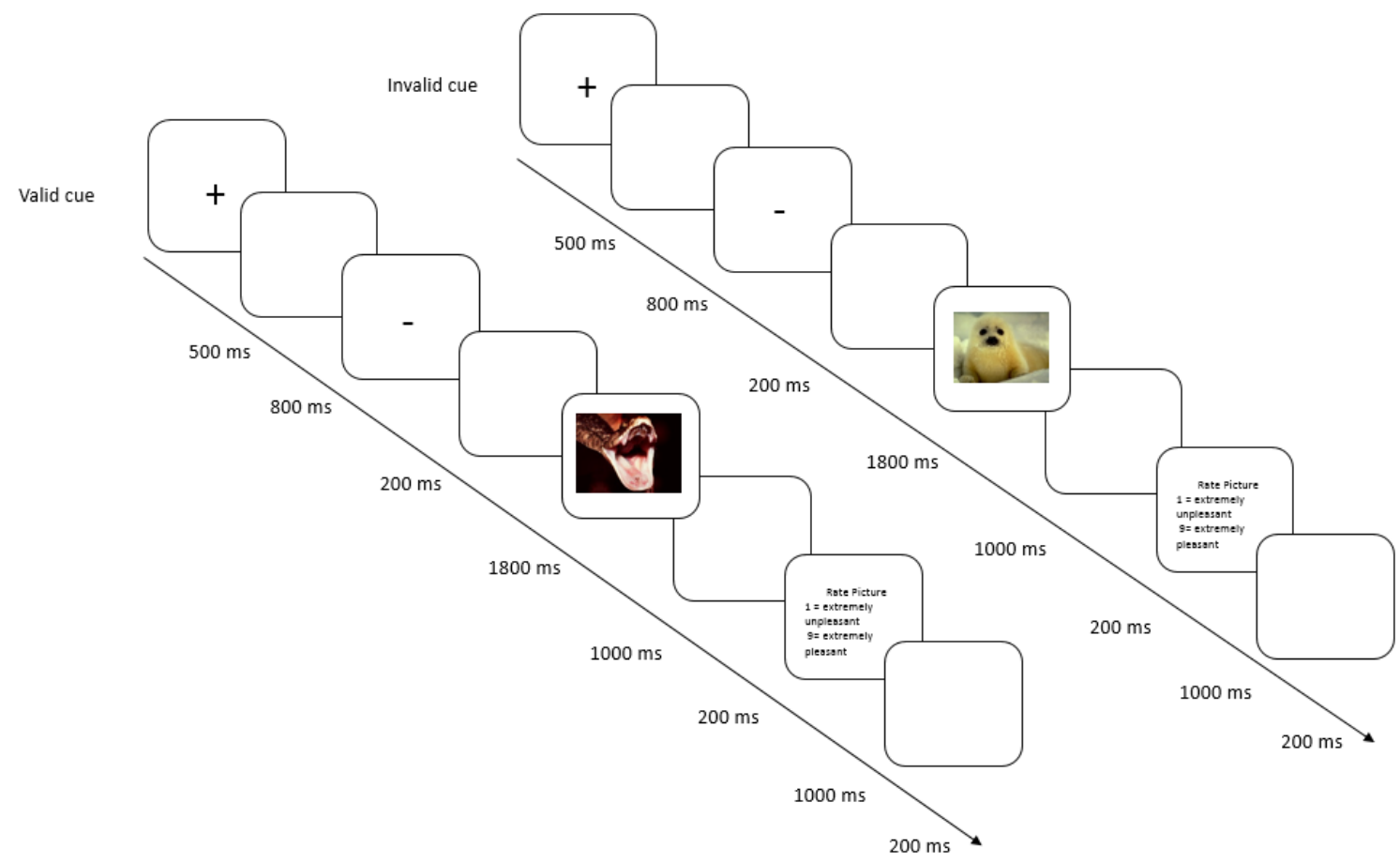

Fig. 1 Schematic illustration of the experimental procedure.

\section{Data Analysis}

The valence ratings of pictures and the response times for these ratings were analysed using 3 x 2 repeated measures ANOVAs with factors Emotion (negative picture, positive picture) and Cue (valid, invalid, and uncertain). Holm-correction (Holm, 1979) was used for follow-up t-tests whenever appropriate.

\section{Results}


Mean valence ratings are displayed in Figure 2. A 3 x 2 repeated measures ANOVA revealed a significant main effect of Emotion $\left(F(1,23)=821.72, p<.001, \eta_{p}{ }^{2}=0.97\right)$, with positive pictures rated higher than negative pictures . The main effect of Cue was not significant $\left(F(2,46)=0.14, p=.870, \eta_{p}{ }^{2}=0.06\right)$. The interaction between Emotion and Cue was significant $\left(F(2,46)=4.85, p=.012, \eta_{p}{ }^{2}=0.17\right)$. To investigate the interaction effect, one-way ANOVAs were conducted on the Cue effect at each level of the factor Emotion. For positive pictures there was no main effect of Cue $\left(F(2,36)=2.30, p=.125, \eta_{p}{ }^{2}=0.09\right)$; for negative pictures there was a significant main effect of Cue $\left(F(2,46)=3.26, p=.048, \eta_{p}{ }^{2}=\right.$ 0.12). Follow-up t-tests revealed a significant difference between valid and invalidly cued negative pictures, with validly cued negative pictures leading to lower valence ratings compared to invalidly cued negative pictures $(t(23)=2.63, p=.045, d=0.15)$. There was no difference between the validly and uncertainly cued $(t(23)=1.01, p=.321, d=0.07)$, or between the invalidly and uncertainly cued negative pictures $(t(23)=1.51, p=.292, d=$ $0.09)$.

\section{A Positive Pictures B Negative Pictures}

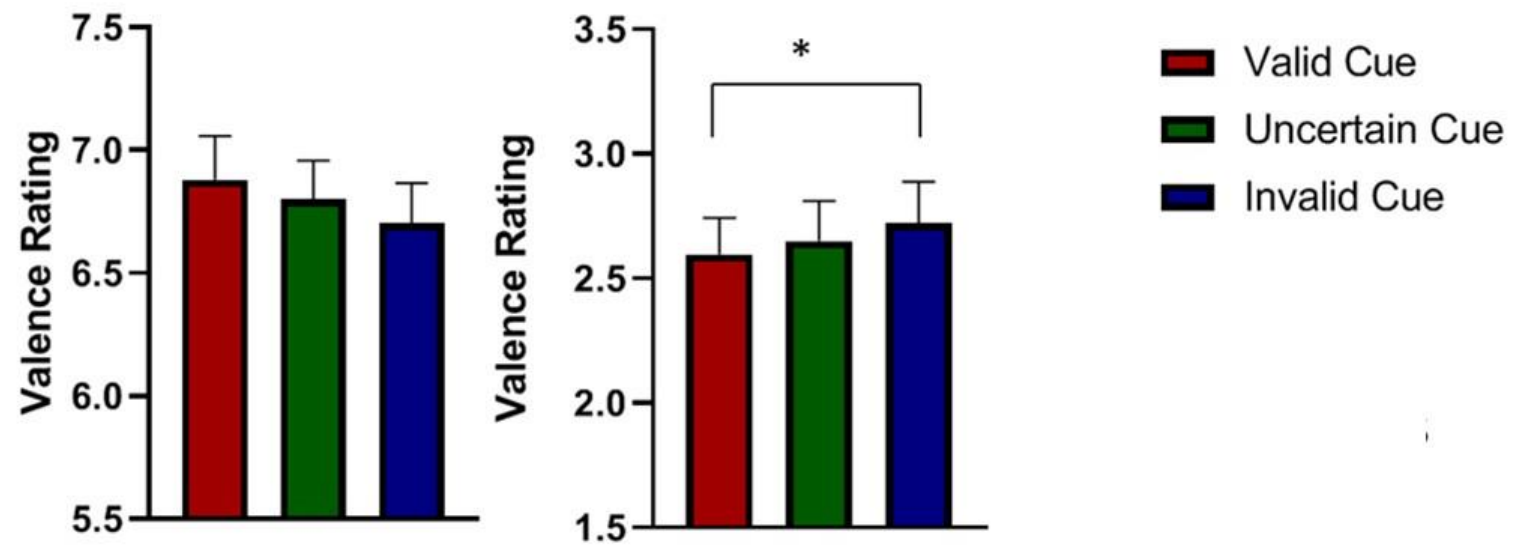


Fig. 2 Valence ratings for (A) positive and (B) negative pictures for the three cue conditions. Error bars indicate standard error of the mean. $* p<.05$

\section{Reaction times}

Descriptive statistics for reaction times are shown in Table 2. There was no main effect of Emotion $\left(F(1,11)=0.94, p=.343, \eta_{p}{ }^{2}=0.04\right)$, and no main effect if Cue $(F(2,34)=$ $0.43, p=.601, \eta_{p}{ }^{2}=0.02$ ). The interaction between Emotion and Cue was also not significant $\left(F(2,36)=3.37, p=.056, \eta_{p}^{2}=0.12\right)$.

Table 2 Means (and standard deviations) for reaction times (ms) for positive and negative pictures for the three cue conditions.

\begin{tabular}{llll}
\hline & & Cue & \\
\hline Picture & Uncertain & Valid & Invalid \\
Positive & $1344.19(509.56)$ & $1299.07(456.12)$ & $1423.07(500.29)$ \\
& & & \\
Negative & $1299.37(552.88)$ & $1311.91(585.23)$ & $1247.57(604.17)$ \\
& & & \\
\hline
\end{tabular}




\section{Discussion}

Our results report the novel finding that invalidly cued negative pictures (i.e., positive picture expected, negative picture presented) led to more neutral valence ratings, compared to validly cued negative pictures (negative picture expected, negative picture presented). The findings are in conceptual agreement with those of Lin et al. (2012), who showed that under conditions of expectation certainty (i.e., where the stimulus was presented with prior indication of its valence) the emotional impact of visual scenes was enhanced, compared to under conditions of expectation uncertainty (where the stimulus was presented without prior indication of its valence).

There was no effect of cue validity on the ratings of positive pictures. This was likely due to the lower arousal levels of the positive pictures compared to the negative pictures. This limitation was addressed in Experiment 2.

\section{Experiment 2}

The main aim of Experiment 2 was to investigate the effects of incongruent expectations on neural responses to emotional pictures, as reflected by the picture-locked $\mathrm{P} 2$, $\mathrm{N} 2$ and LPP components. It also aimed to address a limitation of Experiment 1, namely that the positive and negative stimuli differed in arousal. Based on the results reported by Lin, Schulz, \& Straube $(2015,2016)$, we predicted that invalidly cued pictures would evoked more positive early LPP amplitudes, compared to validly cued pictures. We also analysed the later part $(600-800 \mathrm{~ms})$ of the LPP, to test whether expectation congruency influences more in-depth processing of the emotional content of the pictures (Gole et al., 2012; Leutgeb, 2009). For valence ratings, we expected the same pattern of results as Experiment 1, where invalidly cued pictures led to attenuated (i.e., more neutral) valence ratings compared to validly cued pictures. 
A secondary aim of Experiment 2 was to compare neural activity prior to the onset of the pictures, for cues indicating that the upcoming image was positive, versus cues indicating that the picture was negative. We therefore analysed three cue-locked ERP components, namely the P2, the early posterior negativity (EPN), and the stimulus preceding negativity (SPN), as these components have been shown to reflect anticipatory processes related to emotional stimuli (Brunia, van Boxtel, \& Boecker, 2012; Huang, Shang, Dai, \& Ma, 2017; Michalowski, Pané-Farré, Löw, \& Hamm, 2015).

\section{Method}

\section{Participants}

Twenty-five female participants (age: $M=28.4, S D=9.9$ years) took part in the study. All participants reported no history of neurological or psychological disorders, were right handed, and had normal or corrected-to-normal vision.

\section{Stimuli}

Three-hundred and thirty pictures were selected from the IAPS database; half were positive (valence: $M=7.41, S D=0.54$; arousal: $M=5.18, S D=0.88$ ) and half were negative (valence: $M=2.76, S D=0.67$; arousal: $M=5.30, S D=0.75$ ). The two categories differed significantly in valence $(t(303.60)=68.18, p<.001, d=7.62)$, but not in arousal $(t(318)=$ $1.28, p=.167, d=0.16)$. Thirty of the pictures (15 positive and 15 negative) were selected for inclusion in a practice block.

\section{Procedure}

The same paradigm as in Experiment 1 was used, with the only modifications being that the fixation cross was substituted by a fixation point to rule out any potential confusion between the fixation cross and one of the cue symbols. The uncertain cue condition was 
removed to maximise the number of invalid trials. The total number of trials for each participant was: validly cued positive pictures $=105$; invalidly cued positive pictures $=45$; validly cued negative pictures $=105 ;$ invalidly cued negative pictures $=45$.

\section{EEG data acquisition and pre-processing}

EEG data was recorded from 32 scalp electrodes using an Active Two amplifier system (BioSemi, Amsterdam, Netherlands). Electrodes were placed according to the extended 10-20 system (Nuwer et al., 1998). Four further electrodes were positioned above and below the left eye and on the outer canthi of the left and right eyes, to record the vertical and horizontal electrooculogram (VEOG and HEOG, respectively). EEG signals from all channels were acquired with respect to the common mode sense (CMS) electrode with a sampling rate of $512 \mathrm{~Hz}$. The continuous EEG was divided into epochs offline, beginning 500 $\mathrm{ms}$ prior to stimulus onset and ending $1000 \mathrm{~ms}$ after stimulus onset, and the epochs were digitally filtered (zero phase Butterworth band-pass filter, $0.01-25 \mathrm{~Hz}$ ). The $100 \mathrm{~ms}$ prestimulus period was used for baseline correction. EEG artefacts were rejected using the SCADS procedure with standard parameters (Junghöfer, Elbert, Tucker, \& Rockstroh, 2000). This procedure first detected individual channel artefacts, then transformed the data to the average reference and subsequently identified global artefacts. Epochs that contained more than 10 unreliable electrodes were excluded from analysis on the basis of the distribution of their amplitude, standard deviation and gradient. For the remaining epochs, data from artefact-contaminated sensors was replaced by a statistically weighted spherical interpolation using the complete electrode set. With respect to the spatial distribution of the approximated electrodes, it was ensured that the rejected channels were not localised within one region of the scalp, as this would make interpolation for this area unreliable. Therefore the standard deviation of the spherical splines used for approximation was computed for each epoch and 
epochs that represented outliers from this distribution were rejected. Across all participants the procedure rejected an average of $19.2 \%$ of epochs as artefacts.

\section{Event-related potentials (ERPs)}

Cue-locked ERPs were averaged separately for the positive and negative cue conditions, resulting in two ERPs per participant and electrode. The P2 component was maximal over fronto-central electrode sites (Fz, FC1, FC2, and $\mathrm{Cz}$ ) around $200 \mathrm{~ms}$ after cue onset, and was analysed in a $40 \mathrm{~ms}$ time-window around the peak $(180-220 \mathrm{~ms})$, consistent with previous studies (e.g., Harrison \& Ziessler, 2016; Sheng et al., 2016). The Early Posterior Negativity (EPN) was maximal around 300 ms over occipito-parietal regions (O1, $\mathrm{O} 2, \mathrm{Oz}, \mathrm{PO} 3, \mathrm{PO} 4, \mathrm{P} 7$, and $\mathrm{P} 8$ ) and was analysed in a $80 \mathrm{~ms}$ time window around the peak (260 - $340 \mathrm{~ms}$ ), in close agreement with previous studies (e.g., Schupp et al., 2006; Van Strien et al., 2014). The SPN was analysed at fronto-central electrodes (F3, Fz, F4, FC1, FC2, $\mathrm{C} 3, \mathrm{Cz}$, and C4) between $1200-1600 \mathrm{~ms}$ based on visual inspection and in close agreement with previous studies (e.g., Lin, Gao, et al., 2014).

Picture-locked ERPs were averaged based on the emotional valence of the pictures (positive and negative) and cue conditions (valid and invalid), resulting in four ERPs per participant and electrode. The P2 component was maximal around $150 \mathrm{~ms}$ over fronto-central electrodes, consistent with previous studies (e.g. Lin et al., 2012; Lin et al., 2015). Based on visual inspection of the grand-averaged waveform, the P2 was analysed at fronto-central electrodes (Fz, FC1, FC2, and Cz), using a 40 ms time-window around the peak $(130-170$ ms), in line with previous research (e.g., Lin et al., 2012; Lin et al., 2015). The N2 component was maximal around $250 \mathrm{~ms}$ and was analysed at fronto-central electrodes $(\mathrm{C} 3, \mathrm{C} 4, \mathrm{FC} 1$, FC2, F3, and F4), using a $100 \mathrm{~ms}$ time-window around the peak $(200-300 \mathrm{~ms})$, based on visual inspection and in close agreement with previous studies (e.g. Lin et al., 2012; Yang et 
al., 2010; Harrison et al., 2015; Harrison \& Ziessler, 2016). The Early Posterior Negativity (EPN) was analysed from mean activity in a 200 - $300 \mathrm{~ms}$ time-window at occipito-parietal (O1, O2, Oz, PO3, PO4) electrode locations (Harrison \& Chassy, 2019; Van Strien et al., 2009, 2014). The LPP was analysed at centro-parietal electrodes (CP1, CP2, and Pz) in 200 $\mathrm{ms}$ time windows for the early $(400-600 \mathrm{~ms})$ and late $(600-800 \mathrm{~ms})$ portions, based on visual inspection, and in agreement with previous studies (e.g. Gole et al., 2012; Harrison \& Chassy, 2019; Schindler, Wegrzyn, Setppacher, \& Kissler, 2015). The mean amplitudes averaged across these time-windows and electrode clusters were submitted to 2 x 2 repeatedmeasures ANOVAs with the factors Emotion (positive picture, negative picture) and Cue Validity (valid cue, invalid cue).

\section{Results}

\section{Behavioral Data}

Valence ratings for one participant were excluded as they were an extreme outlier (more than 3 SDs from the mean). A 2 x 2 repeated measures ANOVA was conducted on the ratings with the factors Emotion (positive picture, negative picture) and Cue Validity (valid cue, invalid cue). Mean valence ratings are displayed in Figure 3. There was a significant main effect of Emotion $\left(F(1,23)=504.152, p<.001, \eta_{p}{ }^{2}=0.956\right)$, with higher valence ratings for positive compared to negative pictures. The main effect of Cue Validity was not significant $\left(F(1,23)=1.81, p=.192, \eta_{p}{ }^{2}=0.073\right)$. The interaction between Emotion and Cue Validity was significant $\left(F(1,23)=7.55, p=.011, \eta_{p}{ }^{2}=0.247\right)$.

Follow-up t-tests revealed that for positive pictures $(t(23)=2.56, p=.036, d=0.36)$ invalidly cued pictures had reduced valence ratings compared to validly cued positive pictures. For negative pictures, however, invalidly cued pictures had increased valence ratings compared to validly cued negative pictures $(t(23)=2.43, p=.036, d=0.27)$. 


\section{A Positive pictures B Negative pictures}

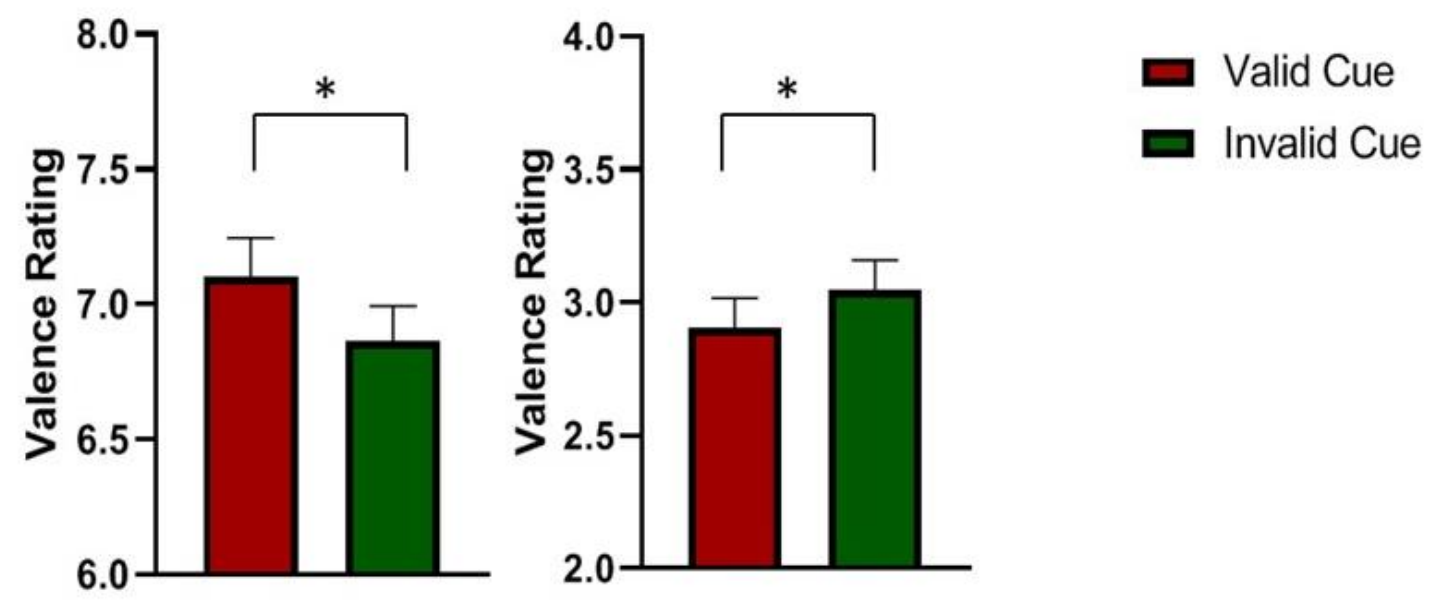

Fig. 3 Valence ratings for positive (A) and negative (B) pictures for each cue validity condition. Error bars indicate standard error of the mean. ${ }^{*} p<.05$

Descriptive statistics for reaction times are shown in Table 3. A 2 x 2 repeated measures ANOVA showed that neither Emotion $\left(F(1,18)=0.10, p=.755, \eta_{p}^{2}=0.01\right)$, nor Cue Validity $\left(F(1,18)=1.13, p=.303, \eta_{p}{ }^{2}=0.06\right)$, nor the interaction between Emotion and Cue Validity $\left(F(1,18)=2.92, p=.105, \eta_{p}{ }^{2}=0.14\right)$ were significant.

Table 3 Means (and standard deviations) for reaction times (ms) for positive and negative pictures for the two cue conditions

\begin{tabular}{lll}
\hline & \multicolumn{2}{c}{ Cue } \\
\hline Picture & Valid & Invalid \\
Positive & $1365.73(725.31)$ & $1558.12(586.28)$ \\
Negative & $1357.07(596.44)$ & $1512.21(675.53)$ \\
\hline
\end{tabular}

Cue-locked ERP results 
A paired-samples t-test indicated that there was a significant difference between the positive and negative cues for the cue-locked P2 component $(t(23)=2.20, p=0.038, d=$ 0.26), with higher activity following cues indicating the future presence of a negative picture compared to those indicating the future presence of a positive picture. The ERP waveform and associated scalp map are shown in Figure 4.

\section{Cue-locked P2 at fronto-central electrodes}
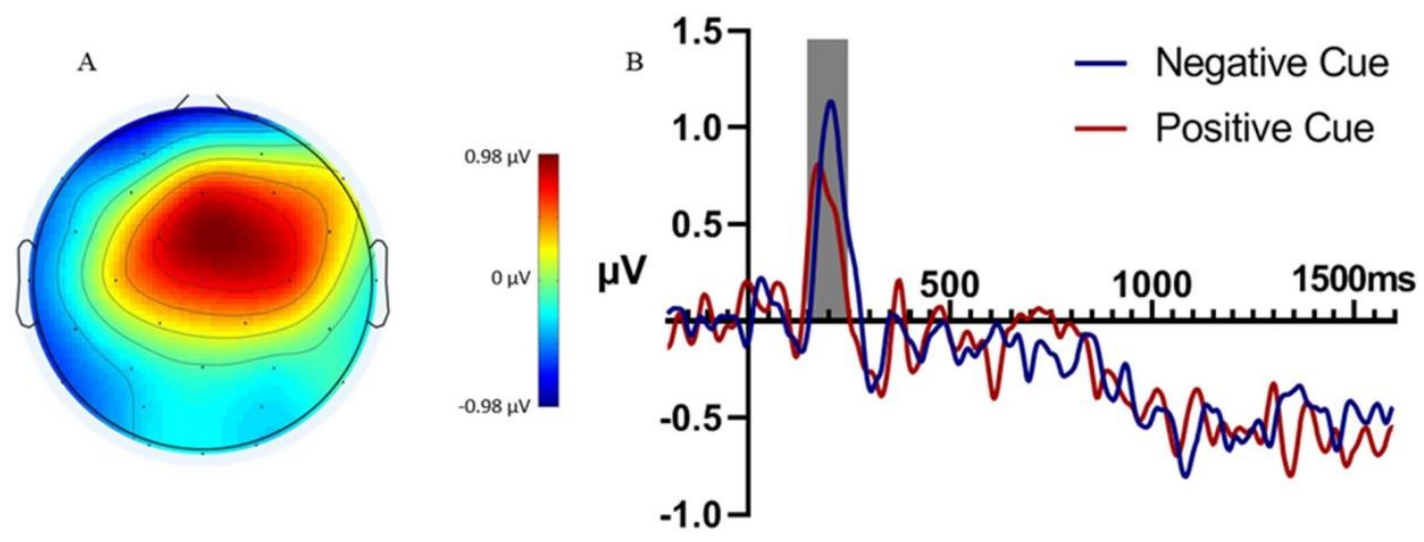

Fig. 4 Scalp map and ERP waveform for the cue-locked P2. A) Scalp map at 200ms after cue onset (peak of P2 component). B) Grand-averaged waveform over midline fronto-central electrodes (Fz, FC1, FC2, and $\mathrm{Cz}$ ), showing the P2 component (shaded area) following positive and negative cues.

There was no significant difference in ERP amplitude between the cue conditions for the $\operatorname{EPN}(t(23)=1.56, p=0.132, d=0.13)$. For the SPN there was no difference in amplitude between the cues $(t(23)=0.73, p=0.474, d=0.11)$. Descriptive statistics are shown in Table 4.

Table 4 Means (and standard deviation) for the cue-locked EPN and SPN amplitudes $(\mu \mathrm{V})$ following positive and negative cues. 


\begin{tabular}{llc}
\hline ERP & Negative Cue & Positive Cue \\
\hline EPN & $-0.10(2.51)$ & $0.25(2.82)$ \\
SPN & $-0.47(1.17)$ & $-0.61(1.24)$ \\
\hline
\end{tabular}

\section{Picture-locked ERP results}

There was a positive peak at fronto-central electrodes $150 \mathrm{~ms}$ after picture onset, consistent with the timing and topography of the P2 component. Statistical analysis of the P2 revealed that there was no main effect of Emotion $\left(F(1,24)=2.96, p=.098, \eta_{p}{ }^{2}=0.110\right)$, no main effect of Cue Validity $\left(F(1,24)=0.839, p=.369, \eta_{p}^{2}=0.040\right)$, and no interaction between Emotion and Cue Validity $\left(F(1,24)=1.47, p=.238, \eta_{p}{ }^{2}=0.058\right)$. Descriptive statistics are presented in Table 5.

A negative peak in the ERPs at fronto-central electrodes around $250 \mathrm{~ms}$ after picture onset, consistent with the N2 component, was observed (see Figure 5). For the N2, there was a main effect of Emotion $\left(F(1,24)=4.81, p=.038, \eta_{p}{ }^{2}=0.167\right)$, with increased amplitude following negative compared to positive pictures. Neither the main effect of Cue Validity $\left(F(1,24)=0.37, p=.547, \eta_{p}{ }^{2}=0.015\right)$, nor the interaction between Emotion and Cue Validity $\left(F(1,24)=0.18, p=.673, \eta_{p}^{2}=0.007\right)$ were significant 
A

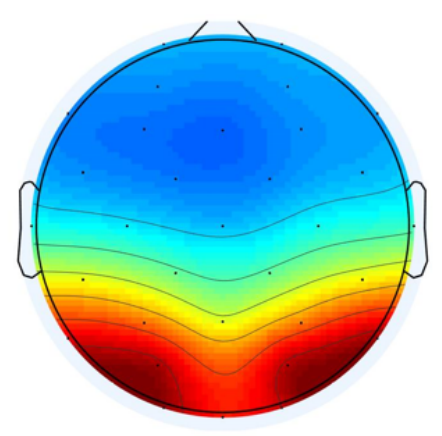

N2 at fronto-central electrodes

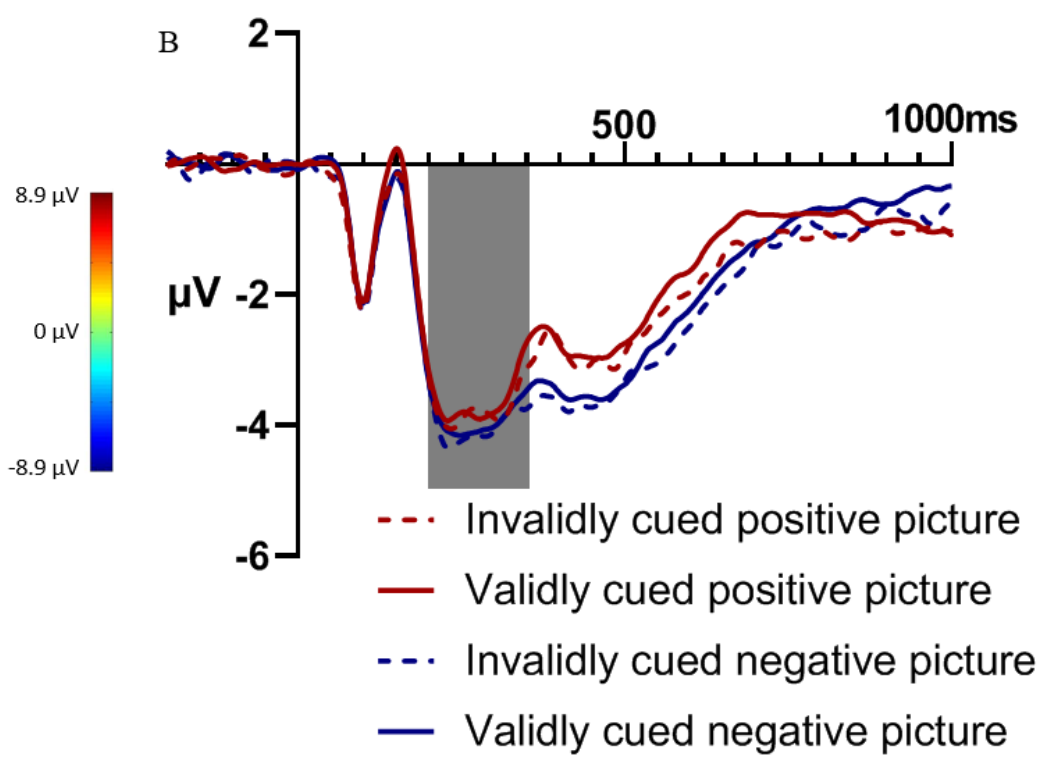

Fig. 5 Scalp map and ERP response of the picture-locked N2 component. A) Scalp map at $250 \mathrm{~ms}$ after picture onset (peak of N2 component). B) Grand-averaged waveform over fronto-central electrodes (FC1, FC2, F3, F4, C3, and C4), showing the N2 component (shaded area) following validly and invalidly cued positive and negative pictures.

The Early Posterior Negativity (EPN) was analysed using a 200 - 300 ms timewindow at occipito-parietal electrode locations (Table 5). There was no main effect of Emotion $\left(F(1,24)=1.88, p=.183, \eta_{p}{ }^{2}=0.073\right)$, no main effect of Cue Validity $(F(1,24)=$ 0.004, $\left.p=.949, \eta_{p}{ }^{2}<0.001\right)$, and no interaction between Emotion and Cue Validity $(F(1,24)$ $\left.=0.450, p=.509, \eta_{p}^{2}=0.018\right)$.

A late positive potential (LPP), consisting of a sustained positive waveform with a peak amplitude at around $520 \mathrm{~ms}$ over centro-parietal electrodes, was observed (Figure 6). Statistical analysis of the early portion of the LPP $(400-600 \mathrm{~ms})$ revealed a significant main effect of Emotion $\left(F(1,24)=11.40, p=.003, \eta_{p}^{2}=0.322\right)$, with increased early LPP amplitude following negative compared to positive pictures. There was also a significant main effect of Cue Validity $\left(F(1,24)=4.59, p=.043, \eta_{p}{ }^{2}=0.161\right)$, with higher amplitudes 
following invalid compared to valid cues. The interaction between Emotion and Cue Validity was not significant $\left(F(1,24)=1.36, p=.289, \eta_{p}^{2}=.054\right)$.

For the later portion of the LPP $(600-800 \mathrm{~ms})$, there was a main effect of Emotion $\left(F(1,24)=18.90, p<.001, \eta_{p}{ }^{2}=0.44\right)$, with increased amplitude following negative compared to positive pictures. There was no main effect of Cue Validity $(F(1,24)=0.24, p=$ $\left..630, \eta_{p}{ }^{2}=0.010\right)$, and the interaction between Emotion and Cue Validity $(F(1,24)=0.88, p=$ $\left..358, \eta_{p}{ }^{2}=0.035\right)$ was not significant. Descriptive statistics can be found in Table 5.
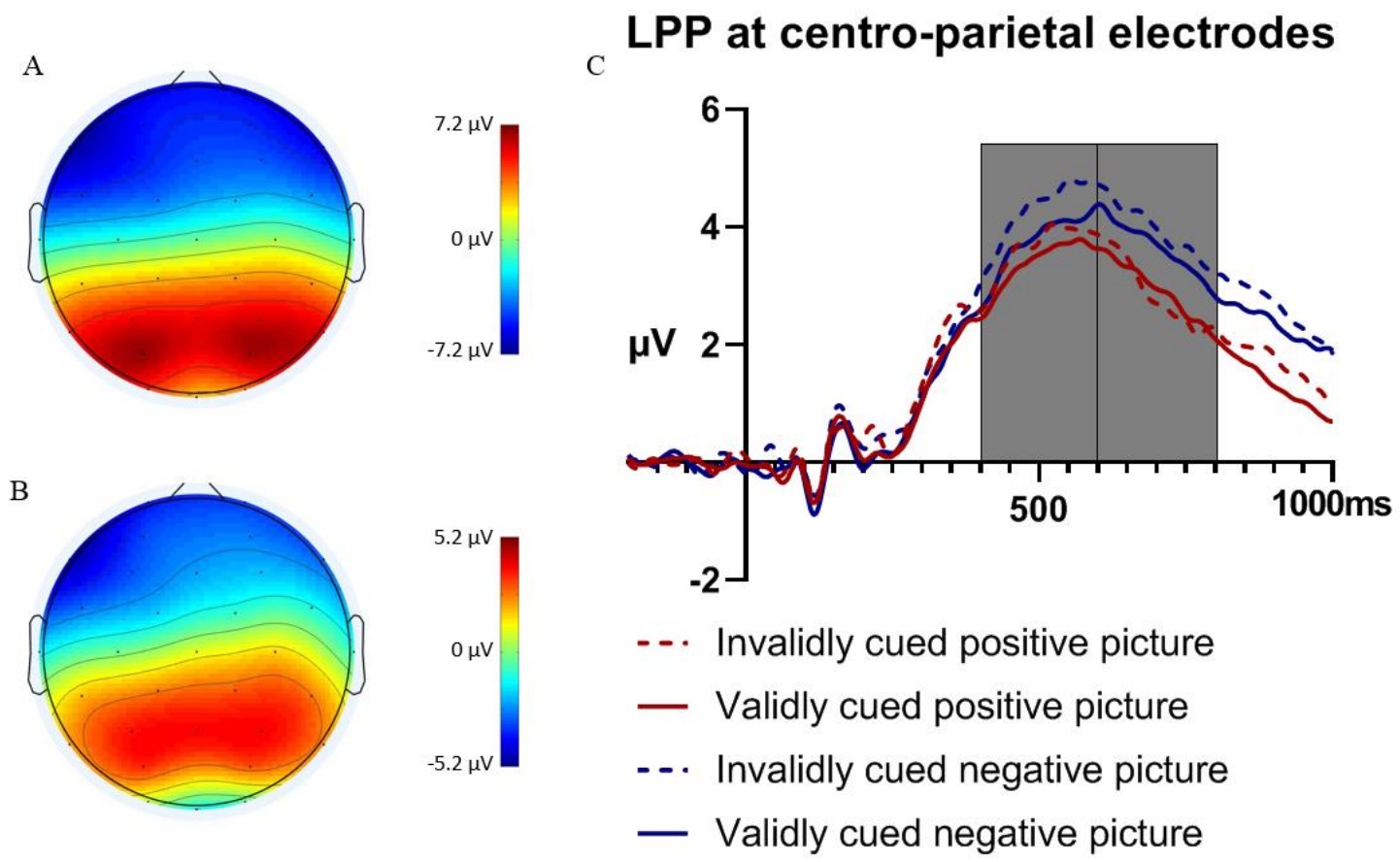

Fig. 6 Scalp map and ERP response of the early and late picture-locked LPP. A. Scalp map at $500 \mathrm{~ms}$ after picture onset (early LPP). B. Scalp map at $700 \mathrm{~ms}$ after picture onset (late LPP). C. Grand-averaged waveform over centro-parietal electrodes (CP1, CP2, and Pz), showing the early (left shaded area) and later (right shaded area) LPP following validly and invalidly cued positive and negative pictures. 
Table 5 Means (and standard deviations) for the picture-locked P2, N2, EPN, and early and late LPP amplitudes (in $\mu \mathrm{V}$ ) following positive and negative pictures, and valid and invalid cues.

\begin{tabular}{lcccc}
\hline ERP & Positive & Negative Picture & Valid Cue & Invalid Cue \\
& Picture & & \\
\hline P2 & $.04(2.73)$ & $-0.43(2.59)$ & $-0.05(2.60)$ & $-0.16(2.73)$ \\
N2 & $-3.78(1.82)$ & $-4.04(1.97)$ & $-3.87(1.86)$ & $-3.95(1.83)$ \\
EPN & $8.10(4.54)$ & $8.40(5.05)$ & $8.26(4.84)$ & $8.25(4.75)$ \\
Early LPP & $3.49(1.98)$ & $3.98(2.33)$ & $3.54(2.18)$ & $3.93(2.19)$ \\
& & & & \\
Late LPP & $2.96(2.21)$ & $3.86(2.65)$ & $3.35(2.36)$ & $3.47(2.45)$ \\
& & & & \\
\hline
\end{tabular}

\section{Correlational analysis}

In a final analysis stage we computed Pearson's correlations between the a) invalid-valid early LLP amplitude difference, and b) invalid-valid valence ratings difference scores, separately for positive and negative images. For positive pictures, the correlation was not significant $(r=.24, p=.264)$, and for negative pictures the correlation was also not significant $(r=.28, p=.192)$.

\section{Discussion}

Behavioural results showed that invalidly cued pictures elicited more neutral valence ratings compared to validly cued pictures, and the electrophysiological results showed an increased amplitude in the early portion $(400-600 \mathrm{~ms})$ of the LPP for invalidly compared to validly cued pictures. There were no effects of cue validity for the P2, N2 and EPN components, nor for the later portion of the LPP. Furthermore, negative pictures were 
associated with an increase in the N2 and LPP (early and late), but there was no effect of emotion for the P2 and EPN components. For the cue-locked ERPs, the P2 was increased following negative compared to positive cues, but there were no differences between cues for the EPN and SPN. We found no response time differences between cue conditions, which is not surprising given that participants were not instructed to respond as quickly as possible when making the valence judgements.

\section{General discussion}

In everyday life, it is unfortunately quite common for our expectations about the emotional quality of an upcoming event to be incorrect. For example, we may predict that seeing a particular person or scene will lead to a pleasurable feeling, when in fact the opposite turns out to be the case. However, relatively little is known about the consequences of emotion expectations in these cases. The current studies aimed to investigate the effects of valid (i.e., where the stimulus valence matched the expected valence) and invalid (i.e., where the stimulus valence was of the opposite valence to the expected valence) expectations on the behavioural (Experiment 1) and electrophysiological (Experiment 2) responses to emotional scenes. Results showed that invalidly cued pictures elicited more attenuated (i.e., less negative for negative pictures, and less positive for positive pictures) valence ratings, and increased amplitude of the early part of the LPP, compared to validly cued pictures.

To the best of our knowledge, the only previous experiment to investigate the effects of invalid expectations on emotional processing of scenes was by Osman and colleagues (2017). However, as valence ratings were not measured, the effects of cue validity on their participants' subjective responses could not be determined. Here, in Experiment 1, we reported the novel finding that cue validity influenced the emotional responses to the images, and that this effect depended on the valence of the images. Invalidly cued negative pictures 
were associated with less negative valence ratings compared to validly cued negative pictures. In other words, when participants were expecting to see a negative stimulus and the stimulus valence was congruent with their expectations, they rated the stimulus as more negative, compared to when they were expecting to view a positive stimulus and instead they encountered a negative stimulus. This behavioural effect was replicated in Experiment 2. These findings are broadly in alignment with the results from Lin and colleagues (Lin et al., 2012; Lin et al., 2015), who found that expected negative pictures received more negative valence ratings, compared to unexpected negative pictures (where the valence of the stimulus was unknown in advance). Our findings provide robust evidence that cues which give information about the expected valence of an upcoming stimulus influence the emotional responses to the presented stimulus. Similarly, in Experiment 2 we found that invalidly cued positive pictures led to less positive valence ratings, compared to validly cued positive pictures. In contrast to the 'Costs and Benefits of Expectations' theory (Golub et al., 2009), that predicts that events that differ from their expected valence would elicit an amplified emotional reaction, the behavioural findings in the current experiments instead showed that a discrepancy between the expected and actual valence resulted in an attenuated response, as indexed by more neutral valence ratings.

ERPs were measured in Experiment 2, in addition to valence ratings, to examine modulations of the electrophysiological responses to affective pictures in relation to the validity of the anticipatory cues. We observed no effects of expectations on the amplitude of the P2 component, in line with previous research (Lin et al., 2018). The N2, reflecting stimulus discrimination and classification, was increased for negative pictures, suggesting enhanced attention allocation following exposure to the negative pictures, in agreement with previous research (Olofsson et al., 2008). Uncertain cues, that are presumed to increase motivated attention prior to the presentation of the pictures, have similarly been linked to a 
subsequent increased $\mathrm{N} 2$ response, reflecting increases in attention during the classification process (Lin et al., 2012). In the current experiment, however, participants could not know the validity of the cue before the presentation of the pictures, and as such, there would be no increased motivation during the expectation of the picture and thus stimulus features such as emotion rather than expectations likely drive these early processing stages.

During the later portion $(600-800 \mathrm{~ms})$ of the LPP, which is thought to reflect more in-depth processing of emotional pictures (Gole et al., 2012; Leutgeb, 2009), we found no differences between validly and invalidly cued pictures. However, ERP effects related to cue validity were apparent in the earlier part of the late positive complex (400 - $600 \mathrm{~ms})$, where the amplitude was increased for invalidly compared to validly cued pictures. Invalidly cued stimuli, and unexpected or surprising events, often elicit a positive shift in amplitude over posterior electrodes around the same time as the early part of the LPP (Curran et al., 2001; Delplanque et al., 2005; Fabiani, Gratton, Karis, \& Donchin, 1987; Mangun \& Hillyard, 1991; Volpe et al., 2007), and our results are consistent with studies of facial expressions showing that expectation incongruency increased the amplitude of the P3/LPP compared to expectation congruency (Lin, Schulz, \& Straube, 2015, 2016). The magnitude of this posterior positivity has been linked to the magnitude of prediction error i.e., the magnitude of the difference between an expected and perceived outcome (Philiastides, Biele, Vavatzanidis, Kazzer, \& Heekeren, 2010). Research using an explicit anticipation task where participants were asked to estimate the likelihood of a given emotional stimulus following the cue found that participants overestimated the likelihood of negative pictures in the uncertain condition, which was associated with an increased LPP-like response (Dietrich et al., 2016; 2017; Lin et al., 2018). The ERP results are in contrast to those of Osman et al. (2017), who examined LPP responses to pictures when the expected and actual stimulus valence differed from either neutral to negative, or from negative to neutral. Osman and colleagues reported no effects of 
cue validity, but this could have been due to the invalidly cued images being insufficiently surprising, as the difference between expected and actual valence was relatively small.

A number of different explanations have been proposed in the literature to explain the behavioural and neural effects of affective expectations, including 'the costs and benefits of expectation' theory, motivational priming, and emotion regulation accounts. The current results are overall not in agreement with the predictions made by the 'costs and benefits of expectations' theory (Golub et al., 2009); in fact, this theory would predict the opposite pattern of results to that which was observed for our valence ratings. Therefore, alternative explanations would more parsimoniously explain the results.

According to a motivational priming account, emotional responses are enhanced when elicited in a congruent emotional state (Lang, Bradley, \& Cuthbert, 1997). A prominent example of this is the emotion-modulated startle response, whereby the startle response is increased when participants view negative compared to positive pictures (e.g. Bradley, Codispoti, \& Lang, 2006). Viewing negative pictures is thought to prime defensive mechanisms and inhibit activation of the appetitive system. In the context of the current experiments, according to this viewpoint the cues should prime the associated defensive or appetitive system, leading to an enhanced emotional response to pictures following valid (i.e., emotionally congruent) cues, and an attenuated response to pictures following invalid (i.e., emotionally incongruent) cues. Our behavioural findings are in line with this account. On the other hand, the neurophysiological responses appear to be inconsistent with a motivational priming account, as the early LPP amplitude was increased in the invalidly cued (i.e., emotionally incongruent) condition. However, it should be noted that the increased early LPP amplitude in the invalid condition may have reflected expectation violation/prediction error processes rather than emotional processing, as discussed previously. Furthermore, according to the motivational priming account it could be expected that there would be differences in 
cue-locked neural activity, reflecting differences in emotional state elicited by the cues. Our cue-locked ERP results offer partial support for this account, as we observed an increased amplitude of the cue-locked P2 for negative cues compared to positive cues, likely reflecting increased attention allocation to cues signalling the imminent appearance of a negative picture. However, we found no amplitude difference between positive and negative cues for the later EPN component, nor for the SPN, which reflects preparation to process the upcoming image.

It is also possible that expectations elicited by the cues influenced emotional processing of the pictures through some type of implicit emotion regulation process. Cognitive reappraisal, i.e., reinterpreting emotional content to change its affective impact (see Gross, 1998, 2001; Ochsner \& Gross, 2005), could potentially provide a plausible explanation for the behavioural results, as expectations based on the cue information may have altered participants' emotional interpretations of the pictures. Invalidly cued positive pictures may have been reframed in a more negative way due to the expectation of viewing a negative picture (and vice-versa for the invalidly cued negative pictures), thus leading to more neutral valence ratings. However, cognitively reinterpreting an image in a way that reduces its emotional impact generally leads to a decreased LPP amplitude (e.g., Foti \& Hajcak, 2008; Hajcak \& Nieuwenhuis, 2006; MacNamara, Foti, \& Hajcak, 2009), therefore this explanation seems somewhat at odds with the increased early LPP amplitude in the invalid compared to valid conditions. In light of these theoretical considerations, future research should attempt to disambiguate the role of motivational priming and cognitive reappraisal on the effects of invalid expectations on emotion processing.

Some potential limitations of the current studies should be acknowledged. Firstly, only female participants were tested, to ensure a more homogenous sample (Galli, Griffiths, \& Otten, 2014; Gole, et al., 2012), as they may be more sensitive to negative (Groen, Wijers, 
Tucha, \& Althaus, 2013) and unanticipated stimuli (Jin et al., 2013; Lin, Liang, et al., 2014). However, this necessarily limits the generalisability of the results. Secondly, participants' mood at the start of the experiment was not measured; this could potentially have affected responses to the emotional material. Thirdly, we did not include neutral pictures in the design, as we were interested in the responses to pictures when the valence of the picture was opposite to the expected valence. The lack of a neutral picture condition precluded investigation into whether the cue validity effect observed in the ERPs was related only to affective stimuli, or more generally to both emotional and non-emotional stimuli.

Nevertheless, this study leaves open a number of avenues for further investigation. Future studies could usefully investigate the effects of anticipation of emotional stimuli on the oscillatory dynamics of neural processing. There is a growing body of evidence confirming the importance of non-phase locked oscillatory activity in the processing of affective cues, and several studies have reported sustained power enhancement in the theta band over posterior electrodes in an early interval following presentation of an affective stimulus (Harrison, Surre, \& Chassy, 2014; Uusberg, Thiruchselvam, \& Gross, 2014). However, it is not currently known whether this early theta rhythm increase is influenced by participants' expectations.

In summary, the current study investigated the effects of valid (i.e., where the stimulus valence matched the expected valence) and invalid (i.e., where the stimulus valence was the opposite valence to the expected valence) expectations on the processing of positive and negative pictures. It was found that invalid expectations led to attenuated subjective responses to emotional pictures and an increased early LPP amplitude. Together, these results show that invalid expectations influence processing of emotional pictures both at the electrophysiological and behavioural levels. 


\section{References}

Bradley, M. M., Codispoti, M., \& Lang, P. J. (2006). A multi-process account of startle modulation during affective perception. Psychophysiology, 43(5), 486-497. https://doi.org/10.1111/j.1469-8986.2006.00412.x

Brunia, C. H. M., van Boxtel, G. J. M., \& Böcker, K. B. E. (2012). Negative Slow Waves as Indices of Anticipation: The Bereitschaftspotential, the Contingent Negative Variation, and the Stimulus-Preceding Negativity. In E. S. Kappenman \& S. J. Luck (Eds.), The Oxford Handbook of Event-Related Potential Components (pp. 189-207). New York: Oxford University Press.

Curran, T., Hills, A., Patterson, M.B., \& Strauss, M.E. (2001). Effects of aging on visuospatial attention: An ERP study. Neuropsychologia, 39, 288-301.

Delplanque, S., Silvert, L., Hot, P., \& Sequeira, H. (2005). Event-related P3a and P3b in response to unpredictable emotional stimuli. Biological Psychology, 68, 107-120. doi:10.1016/j.biopsycho.2004.04.006

Dieterich, R., Endrass, T., \& Kathmann, N. (2016). Uncertainty is associated with increased selective attention and sustained stimulus processing. Cognitive, Affective, \& Behavioral Neuroscience, 16(3), 447-456. https://doi.org/10.3758/s13415-016-0405-8

Dieterich, R., Endrass, T., \& Kathmann, N. (2017). Uncertainty increases neural indices of attention in obsessive-compulsive disorder. Depression and Anxiety, 34(11), 1018-1028. https://doi.org/10.1002/da.22655

Fabiani, M., Gratton, G., Karis, D., Donchin, E., 1987. Definition, identification, and reliability of measurement of the $\mathrm{P} 300$ component of the event-related brain potential. Advances in Psychophysiology 2, 1-78. 
Foti, D., \& Hajcak, G. (2008). Deconstructing reappraisal: Descriptions preceding arousing pictures modulate the subsequent neural response. Journal of Cognitive Neuroscience, 20(6), 977-988.

Foti, D., Hajcak, G., \& Dien, J. (2009). Differentiating neural responses to emotional pictures: Evidence from temporal-spatial PCA. Psychophysiology, 46(3), 521-530. https://doi.org/10.1111/j.1469-8986.2009.00796.x

Galli, G., Griffiths, V. A., \& Otten, L. J. (2014). Emotion regulation modulates anticipatory brain activity that predicts emotional memory encoding in women. Social Cognitive and Affective Neuroscience, 9(3), 378-384. https://doi.org/10.1093/scan/nss145

Gole, M., Schäfer, A., \& Schienle, A. (2012). Event-related potentials during exposure to aversion and its anticipation: The moderating effect of intolerance of uncertainty. Neuroscience Letters, 507(2), 112-117. https://doi.org/10.1016/j.neulet.2011.11.054 Golub, S.A., Gilbert, D.T., \& Wilson, T.D. (2009). Anticipating one's troubles: the costs and benefits of negative expectations. Emotion, 9, 277-281.

Groen, Y., Wijers, A. A., Tucha, O., \& Althaus, M. (2013). Are there sex differences in ERPs related to processing empathy-evoking pictures? Neuropsychologia, 51(1), 142-155. https://doi.org/10.1016/j.neuropsychologia.2012.11.012

Gross, J. J. (1998). The emerging field of emotion regulation: an integrative review. Review of General Psychology, 2(3), 271.

Gross, J. J. (2001). Emotion regulation in adulthood: Timing is everything. Current Directions in Psychological Science, 10(6), 214-219.

Hajcak, G., MacNamara, A., \& Olvet, D. M. (2010). Event-Related Potentials, Emotion, and Emotion Regulation: An Integrative Review. Developmental Neuropsychology, 35(2), 129155. https://doi.org/10.1080/87565640903526504 
Hajcak, G., \& Nieuwenhuis, S. (2006). Reappraisal modulates the electrocortical response to unpleasant pictures. Cognitive, Affective, \& Behavioral Neuroscience, 6(4), 291-297.

Harrison, N.R., \& Chassy, P. (2019). Habitual use of cognitive reappraisal is associated with decreased amplitude of the late positive potential (LPP) elicited by threatening pictures. Journal of Psychophysiology, 33(1), 22-31.

Harrison, N.R., Surre, F., \& Chassy, P. (2014). Event-related EEG theta-band oscillatory responses to threatening pictures: Modulation by spontaneous emotion regulation strategy. Perception ECVP abstract, 43, 135.

Harrison, N. R., Witheridge, S., Makin, A., Wuerger, S. M., Pegna, A. J., \& Meyer, G. F. (2015). The effects of stereo disparity on the behavioural and electrophysiological correlates of perception of audio-visual motion in depth. Neuropsychologia 78, 51-62.

Harrison, N.R., \& Ziessler, M. (2016). Effect anticipation affects perceptual, cognitive, and motor phases of response preparation: evidence from an event-related potential (ERP) study. Frontiers in Human Neuroscience, 10:5. doi:10.3389/fnhum.2016.00005.

Holm, S. (1979). A Simple Sequentially Rejective Multiple Test Procedure. Scandinavian Journal of Statistics, 6, 65-70.

Huang, Y., Shang, Q., Dai, S., \& Ma, Q. (2017). Dread of uncertain pain: An event-related potential study. PLoS ONE 12(8): e0182489.

Jin, Y., Yan, K., Zhang, Y., Jiang, Y., Tao, R., \& Zheng, X. (2013). Gender differences in detecting unanticipated stimuli: An ERP study. Neuroscience Letters, 538(Supplement C), 38-42. https://doi.org/10.1016/j.neulet.2013.01.020

Junghöfer, M., Elbert, T., Tucker, D. M., \& Rockstroh, B. (2000). Statistical control of artifacts in dense array EEG/MEG studies. Psychophysiology, 37, 523-532.

Lang, P. J., Bradley, M. M., \& Cuthbert, B. N. (1997). Motivated attention: Affect, activation, and action. In P. J. Lang, R. F. Simons, \& M. T. Balaban (Eds.), Attention and 
orienting: Sensory and motivational processes (pp. 97-135). Mahwah, NJ, US: Lawrence Erlbaum Associates Publishers.

Lang, P. J., Bradley, M. M., \& Cuthbert, B. N. (2008). International affective picture system (IAPS): Affective ratings of pictures and instruction manual. Technical Report A-8. University of Florida, Gainesville, FL.

Leutgeb, V., Schäfer, A., \& Schienle, A. (2009). An event-related potential study on exposure therapy for patients suffering from spider phobia. Biological Psychology, 82, 293-300.

Lin, H., Gao, H., Ye, Z., Wang, P., Tao, L., Ke, X., ... Jin, H. (2012). Expectation enhances event-related responses to affective stimuli. Neuroscience Letters, 522(2), 123-127. https://doi.org/10.1016/j.neulet.2012.06.022

Lin, H., Jin, H., Liang, J., Yin, R., Liu, T., \& Wang, Y. (2015). Effects of Uncertainty on ERPs to Emotional Pictures Depend on Emotional Valence. Frontiers in Psychology, 6. https://doi.org/10.3389/fpsyg.2015.01927

Lin, H., Liang, J., Jin, H., \& Zhao, D. (2018). Differential effects of uncertainty on LPP responses to emotional events during explicit and implicit anticipation. International Journal of Psychophysiology, 129, 41-51. https://doi.org/10.1016/j.ijpsycho.2018.04.012 Lin, H., Liang, J., Xie, W., Li, S., Xiang, J., Xu, G., ... Jin, H. (2014). Sex differences in the modulation of emotional processing by expectation: NeuroReport, 25(12), 938-942.

\section{https://doi.org/10.1097/WNR.0000000000000215}

Lin, H., Schulz, C., \& Straube, T. (2015). Cognitive tasks during expectation affect the congruency ERP effects to facial expressions. Frontiers in Human Neuroscience, 9:596. doi: 10.3389/fnhum.2015.00596

Lin, H., Schulz, C., \& Straube, T. (2016). Effects of expectation congruency on event-related potentials (ERPs) to facial expressions depend on cognitive load during the expectation phase. Biological Psychology, 120, 126-136. 
MacNamara, A., Foti, D., \& Hajcak, G. (2009). Tell me about it: Neural activity elicited by emotional pictures and preceding descriptions. Emotion, 9(4), 531-543.

\section{https://doi.org/10.1037/a0016251}

Mangun, G. R., \& Hillyard, S. A. (1991). Modulations of sensory-evoked brain potentials indicate changes in perceptual processing during visual-spatial priming. Journal of Experimental Psychology: Human Perception and Performance, 17, 1057-1074.

Mellers, B. A., Schwartz, A., Ho, K., \& Ritov, I. (1997). Decision affect theory: Emotional reactions to the outcomes of risky options. Psychological Science, 8, 423-429.

Michalowski, J. M., Pané-Farré, C. A., Löw, A., \& Hamm, A. O. (2015). Brain dynamics of visual attention during anticipation and encoding of threat- and safe-cues in spider-phobic individuals. Social Cognitive and Affective Neuroscience, 10(9), 1177-1186. https://doi.org/10.1093/scan/nsv002

Nuwer, M. R., Comi, G., Emerson, R., Fuglsang-Frederiksen, A., Guérit, J. M., Hinrichs, H., ... Rappelsburger, P. (1998). IFCN standards for digital recording of clinical EEG.

International Federation of Clinical Neurophysiology. Electroencephalography and Clinical Neurophysiology, 106(3), 259-261.

Ochsner, K., \& Gross, J. (2005). The cognitive control of emotion. Trends in Cognitive Sciences, 9(5), 242-249. https://doi.org/10.1016/j.tics.2005.03.010

Olofsson, J. K., Nordin, S., Sequeira, H., \& Polich, J. (2008). Affective picture processing: An integrative review of ERP findings. Biological Psychology, 77(3), 247-265. https://doi.org/10.1016/j.biopsycho.2007.11.006

Osman, A., Paczynski, M., \& Jha, A. P. (2017). Affective expectations influence neural responses to stressful images in soldiers. Military Psychology, 29(1), 41-57. https://doi.org/10.1037/mil0000128 
Philiastides, M. G., Biele, G., Vavatzanidis, N., Kazzer, P., \& Heekeren, H. R. (2010). Temporal dynamics of prediction error processing during reward-based decision making. NeuroImage, 53(1), 221-232. https://doi.org/10.1016/j.neuroimage.2010.05.052 Schindler, S., Wegrzyn, M., Steppacher, I., \& Kissler, J. (2015). Perceived communicative context and emotional content amplify visual word processing in the fusiform gyrus. The Journal of Neuroscience: The Official Journal of the Society for Neuroscience, 35(15), 60106019. https://doi.org/10.1523/JNEUROSCI.3346-14.2015

Uusberg, A., Thiruchselvam, R., \& Gross, J.J. (2014). Using distraction to regulate emotion: Insights from EEG theta dynamics. International Journal of Psychophysiology, 91, 254-260. Volpe,U.,Mucci,A.,Bucci,P.,Merlotti,E.,Galderisi,S.,\& Maj, M.(2007). The cortical generators of P3a and P3b: a LORETA study. Brain Research Bulletin, 73, 220-230. doi:10.1016/j.brainresbull.2007.03.003 